\title{
Direct Peritoneal Resuscitation Improves Mesenteric Perfusion by Nitric Oxide Dependent Pathways
}

Sina Khaneki MD, Amanda R. Jensen MD, Natalie A. Drucker MD, Troy A. Markel MD

\author{
Department of Surgery, Section of Pediatric Surgery \\ Indiana University School of Medicine,
}

Riley Hospital for Children and Indiana University Health

Indianapolis, IN 46202

\begin{abstract}
SK performed animal I/R experiments and drafted the manuscript, SK \& NAD performed histological grading and statistical analysis, SK performed protein isolation and bioplex analysis, ARJ prepared histology slides, TAM contributed critical ideas, assistance and manuscript advice. All authors provided critical revisions to the manuscript and assisted with its final preparation
\end{abstract}

This publication was made possible with support from:

1) KL2TR001106 (A. Shekhar, PI) from the National Institutes of Health, National Center for Advancing Translational Sciences, Clinical and Translational Sciences Award

2) Indiana University Health, Indianapolis, IN

\section{Corresponding Author:}

Troy A. Markel, MD

Riley Hospital for Children

705 Riley Hospital Dr.

$\mathrm{RI} 2500$

Indianapolis, IN 46202

tmarkel@iupui.edu

This is the author's manuscript of the article published in final edited form as:

Khaneki, S., Jensen, A. R., Drucker, N. A., \& Markel, T. A. (2017). Direct Peritoneal Resuscitation Improves Mesenteric Perfusion by Nitric Oxide Dependent Pathways. Journal of Surgical Research. 


\section{ABSTRACT}

Introduction: Direct peritoneal resuscitation (DPR) has been shown to increase survival following intestinal ischemia and reperfusion injury (I/R). We have previously appreciated that minimal essential medium (MEM), a synthetic cell culture medium with bovine serum, glutamine, and antibiotics, contributes to these benefits. We hypothesized that:

1) DPR using MEM as a dialysate would increase mesenteric perfusion, improve intestinal mucosal injury, and limit intestinal and hepatic inflammation following intestinal $\mathrm{I} / \mathrm{R}$, and 2) these improvements would be dependent on endothelial nitric oxide pathways.

\section{Methods:}

Eight-week-old C57BI6J wild type (WT) and eNOS Knock Out (eNOSKO) male mice were anesthetized and intestinal ischemia was induced for 60 minutes. Following ischemia, $1 \mathrm{ml}$ of PBS vehicle or MEM was injected into the abdominal cavity. Intestinal perfusion was reassessed after $48 \mathrm{~h}$. Animals were then euthanized and intestines and livers explanted for histologic and molecular analysis.

\section{Results:}

DPR with MEM significantly improved mesenteric perfusion compared to vehicle (PBS) as measured by Laser Doppler Imaging (WT+MEM $91.58 \pm 13.74 \%$, WT+Vehicle $44.27 \pm 11.93 \%, p<0.05)$, however these benefits were lost when endothelial nitric oxide signaling pathways were ablated (eNOS KO+MEM $21.72 \pm 5.67 \%$, eNOSKO+Vehicle $45.24 \pm 11.31 \%)$. Wild type mice treated with MEM also had significantly better preservation of their mucosal architecture (WT+MEM Mdn=1.0, IQR=1.25, WT+Vehicle $M d n=3.0, I Q R=2.0, p<0.05)$. When we compared eNOS KO mice treated with either MEM or vehicle the protective effect of MEM disappeared (eNOS KO+MEM Mdn=2.0, $I Q R=2.25$, eNOS $K O+V e h i c l e ~ M d n=2.0, I Q R=1.0 p>0.05)$. Intestinal levels of IL-1 $\beta$ were 
increased in WT animals treated with MEM as compared to eNOSKOs, while concentrations of intestinal IL-6 were similar between groups. Hepatic levels of both IL$1 \beta$ and IL-6 were significantly elevated in eNOSKOs compared to WT treated with MEM. Conclusion: Direct peritoneal resuscitation with MEM has significant therapeutic potential for improving mesenteric perfusion, intestinal injury, and the local inflammatory response following intestinal I/R. These benefits appear to be dependent on nitric oxide signaling within the endothelium.

\section{KEYWORDS:}

Direct peritoneal resuscitation, ischemia and reperfusion, endothelial nitric oxide synthase, Superior Mesenteric Artery, Intestinal Ischemia, perfusion

\section{INTRODUCTION:}

Acute intestinal ischemia is a highly morbid condition that may result in the need for emergent surgical intervention. The acute inflammatory response associated with this condition may lead to multisystem organ failure or death. Survivors may require lifelong dependence on total parenteral nutrition, particularly if a large amount of small bowel is removed secondary to necrosis [1]. The superior mesenteric artery (SMA) supplies blood to most of the small intestine and proximal large intestine [2]. This artery is most frequently involved in intestinal vascular events. Emboli or thromboses within this vessel can result in mortality as high as $65 \%$ even with treatment [3]. At present most therapeutic interventions focus on resolving the acute thrombus and restoring blood flow to the ischemic bowel. However, even after restoration of blood flow, morbidity and mortality remains quite high [3]. Therefore, alternative strategies that are designed to improve intestinal microvascular bloodflow and limit intestinal damage are needed. 
Previous studies have shown that direct peritoneal resuscitation (DPR) using MEM, a synthetic cell culture medium with bovine serum, glutamine, and antibiotics, improves survival and decreases inflammation within small intestines in an ischemia and reperfusion (I/R) injury model [4]. Peritoneal resuscitation has been utilized for trauma and hypothermic resuscitation, has decreased the time to abdominal closure following damage control laparotomy, and has reduced intrabdominal complications following these trauma related operations[5]. Peritoneal resuscitation has been associated with improved hepatic perfusion, decreased organ edema, and lower levels of circulating proinflammatory cytokines following abdominal injury [6]. Although the definitive molecular mechanisms of DPR therapy have not been elucidated, the protective effects may be associated with decreased local or systemic inflammation [7].

Although ischemic injury is detrimental to the intestine, the associated reperfusion after the thrombus has been removed can provide a "second hit" of injury. After bloodflow is restored, an increase in oxygen to previously ischemic tissue leads to formation of reactive oxygen species, which can cause terminal cellular injuries [8]. Endogenous nitric oxide (NO) is one of the mechanisms by which cells combat reactive oxygen species [9]. Increased production of endogenous NO thorough the endothelial nitric oxide synthase (eNOS) pathway decreases formation of reactive oxygen species, limits the local inflammatory response, and increases vascular dilation [9-11].

Direct peritoneal resuscitation may provide a novel therapeutic opportunity for clinicians to deliver targeted therapies to ischemic tissues in the early post-ischemic state. We have previously observed a tremendous therapeutic benefit with the use of minimal essential cell media (MEM) as a dialysate for DPR following intestinal ischemia. It is not known if eNOS pathways play a role in the intestinal recovery following MEM infusion. We therefore hypothesized that: 1) DPR using MEM as a dialysate would 
increase mesenteric perfusion, improve intestinal mucosal injury, and limit intestinal and hepatic inflammation following intestinal $\mathrm{I} / \mathrm{R}$, and 2) these improvements would be dependent on endothelial nitric oxide pathways.

\section{MATERIALS AND METHODS:}

\section{Murine Model of Intestinal Ischemia and Reperfusion Injury}

The Indiana University Institutional Animal Care and Use Committee approved the experimental protocol and use of animals in this study. Adult male C57BL/6J (Stock No: 00664) and eNOS KO mice (B6.129P2-Nos3tm1Unc/J, Stock No: 002684) between the age of 8 to 12 weeks were purchased from Jackson Laboratory (Bar Harbor, ME) and were allowed to acclimate in animal housing for 48 hours prior to experimentation. They were allowed free access to standard chow and were kept in a 12 hour light-dark cycle.

Animals were divided into 4 groups (WT+Vehicle, WT+MEM, eNOSKO+Vehicle, eNOSKO+MEM, $\mathrm{N}=7 /$ group). Prior to surgery mice were induced with $3 \%$ isoflurane and maintained at $1.5 \%$ isoflurane in oxygen for the duration of the procedure. A heating pad was utilized to achieve homeostatic temperature for the entire duration of intervention as well as during the post anesthesia recovery phase. To restore intraoperative fluid losses, $1 \mathrm{~mL}$ of normal saline was injected subcutaneously prior to start of the procedure. Abdominal hair was removed using hair removal lotion and later prepped with $70 \%$ alcohol and betadine. lodine impregnated dressing (loban) was placed on the skin of the animal to cover the operative filed prior to incision.

A midline laparotomy was performed and the intestines were eviscerated. The SMA was identified and a non-traumatic vascular clamp was placed across the root to occlude the artery. The intestines were then replaced back into the abdominal cavity and skin sutures were placed to prevent evaporative loss of fluid and heat. After 60 minutes of ischemia, the abdominal wall was reopened, the clamp was removed, and direct 
peritoneal resuscitation was initiated using either $1 \mathrm{~mL}$ of Minimum Essential Media ((Life Technologies) supplemented with 16\% fetal bovine serum (Atlanta Biologicals), $1 \%$ penicillin/streptomycin (Sigma-Aldrich), and 1\% glutamine (Sigma Aldrich)) in the intervention group or $1 \mathrm{~mL}$ of Phosphate Buffer Saline (PBS) in the vehicle groups. Analgesia was achieved using bupprenorphine $(1 \mathrm{mg} / \mathrm{kg})$ and carprofen $(5 \mathrm{mg} / \mathrm{kg})$. After completion of surgery animals were placed in a cage without bedding and allowed to recover under observation. Once animals were fully recovered they were returned to the animal housing facility.

\section{Perfusion analysis:}

Perfusion was analyzed using a Laser Doppler perfusion Imager (LDI; Moor Instruments, Wilmington, DE). Images were acquired at baseline, at the initial clamping of the superior mesenteric artery to confirm ischemia, and 48 hours after recovery. A region of interest was created around the entirety of exposed intestines to obtain a flux mean perfusion within this region. Three images were acquired at each time point and averaged. Perfusion data was expressed as a percentage of baseline (mean $\pm S E M)$. After the 48 hour recovery analysis, animals were euthanized with isoflurane overdose and cervical dislocation, and intestinal tissues were explanted for further analysis. Animals that died prior to the 48 hour analysis were given an arbitrary perfusion score of 0.

\section{Histology Scoring:}

Intestines were harvested after 48 hours of recovery. Terminal ileal tissue was fixed in $4 \%$ paraformaldehyde for 24 hours and dehydrated in $70 \%$ ethanol. The tissues were paraffin-embedded and later stained with hematoxylin and eosin. Histology scoring was performed as previously described: 0 , no damage; 1 , subepithelial space at the 
villus tips; 2 , loss of mucosal lining at the villus tips; 3 , loss of less than half of the villus structure; 4 , loss of more than half of the villus structures; and 5, transmural necrosis $[12,13]$. All the histology slides were assessed and scored by two blinded authors (SK, NAD) and scores were expressed as a median with inter-quartile range

\section{Cytokine Analysis:}

Following euthanasia, a portion of mouse intestinal and liver tissues were harvested, snap frozen, and stored at $-80^{\circ} \mathrm{C}$. Tissues were later thawed and homogenized in ice cold RIPA buffer (Sigma, St. Louis, MO) with added protease and phosphatase inhibitor (1:100 dilution, Sigma, St. Louis, MO) using a Bullet Blender (Next Advance, Averill Park, NY). After tissue homogenization, the samples were centrifuged at $12,000 \mathrm{rpm}$ to sediment solid particles and the supernatants were transferred to fresh Eppendorf tubes. Protein concentration in each homogenate was quantified using Bradford assay with a spectrophotometer (VersaMax microplate reader; Molecular Devices, Sunnyvale, CA).

Intestinal and hepatic IL-1 $\beta$ and IL-6 levels were determined by enzyme-linked immunosorbent assay (ELISA) using a commercially available ELISA set (R\&D Systems Inc., Minneapolis, MN). ELISA was performed according to the manufacturer's instructions. All samples and standards were measured in duplicate. Values were then normalized to total protein concentrations in samples. MEM treated groups were then compared between WT and eNOS KO groups by normalizing treated groups to their respective vehicle control. Data are expressed as folds of vehicle.

\section{Statistical analysis}

Statistical power was calculated using Clinclac number calculator (www.clincalc.com) to yield a power of $80 \%$ at alpha level of 0.05 . We anticipated a 
mean perfusion of $50+/-20$ in our wild type and eNOS KO control groups and estimated a 50\% increase in perfusion in wild type mice treated with MEM. These parameters suggested 7 subjects per group.

Continuous variable such as perfusion and cytokine data were reported as mean +/- SEM while ordinal data such as histology scoring was reported as median with interquartile range. All data were non-parametric and compared using Mann-Whitney test in GraphPad Prism 7 (GraphPad Software, La Jolla, CA). A p value of less than 0.05 was considered statistically significant.

\section{RESULTS:}

DPR with MEM Improves Intestinal Perfusion Following I/R Through eNOS Dependent Pathways:

Intestinal perfusion was measured using Laser Doppler Imaging at 48 hours post ischemia. Two animals died prior to perfusion analysis and one animal had necrotic bowel with no flow detected at 48 hours post ischemia. These animals were given an arbitrary perfusion score of 0 . In wild type mice, direct peritoneal resuscitation using MEM led to a significantly higher recovery of mesenteric perfusion at 48 hours following injury (WT+MEM $91.58 \pm 13.74 \%$, WT+Vehicle $44.27 \pm 11.93 \%, \quad p<0.05$, Figure $1 \mathrm{~A})$. However, when eNOS was genetically ablated, there was no notable difference in the recovery of post-ischemic mesenteric perfusion between MEM or vehicle groups. (eNOS KO+MEM $21.72 \pm 5.67 \%$, eNOSKO+Vehicle $45.24 \pm 11.31 \%$, Figure 1B).

DPR with MEM Improves Intestinal Mucosal Injury Following I/R Through eNOS Dependent Pathways: 
In wild type mice, direct peritoneal resuscitation using MEM led to significantly better preservation of intestinal mucosal architecture compared to PBS vehicle $(\mathrm{WT}+\mathrm{MEM} M d n=1.0, \mathrm{IQR}=1.25, \mathrm{WT}+$ Vehicle $M d n=3.0, \mathrm{IQR}=2.0, \mathrm{p}<0.05$ Figure $2 \mathrm{~A})$. However, when eNOS was ablated, no notable differences were seen in the postischemic histological architecture of the small intestine in vehicle or MEM treated groups (eNOS KO+MEM Mdn=2.0, IQR=2.25, eNOS KO+Vehicle $M d n=2.0, I Q R=1.0$, Figure 2B).

\section{Ablation of eNOS Appears to Have a Greater Effect on Hepatic Inflammation Following}

\section{DPR Therapy with MEM}

Levels of the inflammatory markers IL- $1 \beta$ and IL- 6 were assessed as surrogates for inflammation within the intestine. Data are expressed as folds of vehicle. Following DPR therapy with MEM, eNOS ablated animals were found to have lower levels of intestinal IL-1 $\beta$ compared to WT. (eNOSKO 0.73+/-0.10, WT 2.0+/-0.39, $p<0.05$, Figure 3A). No differences in intestinal IL-6 levels were seen between WT and eNOSKO groups with the use of MEM (Figure 3B).

Levels of hepatic IL-1 $\beta$ and IL- 6 were also assessed. Following DPR therapy with MEM, eNOS ablated animals were found to have higher levels of hepatic IL-1 $\beta$ compared to WT. (eNOSKO 1.4+/-0.19, WT 0.87+/-0.05, p<0.05, Figure 4A). eNOS ablated animals were also found to have higher levels of hepatic IL-6 compared to WT following DPR therapy with MEM. (eNOSKO 1.4+/-0.21, WT 0.73+/-0.04, $p<0.05$, Figure 4B).

\section{DISCUSSION:}

DPR may be used as a novel therapy for those with acute intestinal ischemia. Previous studies have shown that DPR improves microcirculatory flow in animal models 
following injury [14]. The mechanism by which blood flow is more readily restored with DPR has not been completely elucidated. Herein we discovered that DPR with MEM improves mesenteric perfusion, intestinal injury, and alters intestinal and hepatic inflammation following injury.

Minimal essential medium (MEM) is a synthetic culture medium with added fetal bovine serum (FBS), glutamine, and antibiotics. It is a standard culture media for in vitro experiments and is used to maintain homeostasis in a number of different cell lines. We previously hypothesized that this dialysate would also portray protective properties in an in vivo model of ischemia. Based on our previous work, we learned that MEM conveyed superior survival benefits compared to either plain (serum free, glutamine free, antibiotic free) media or PBS when used as a dialysate during DPR [4]. Previous work also showed a correlation between increased survival and improved mesenteric perfusion $[15,16]$. Therefore, it stood to reason that the survival benefits to MEM would also be related to improved perfusion.

Endogenous nitric oxide production via the eNOS pathway in endothelial cells is essential to combat free radical injury and prevent cellular apoptosis and swelling in I/R injury [9]. In addition, these pathways are critical for the process of vasodilation [17]. Therefore, given that we noted improved mesenteric perfusion in treated animals within this study, we elected to see if eNOS was important for the observed effects following DPR. We observed a significant increase in the recovery of post-ischemic mesenteric perfusion with the addition of MEM when eNOS was present. However, once it was ablated in the knockout animals, DPR with MEM did not appear to offer any further protection. These results would suggest that the use of MEM as a dialysate for DPR, as well as the resultant improvements in mesenteric perfusion, work through endothelial nitric oxide dependent pathways.

In addition, the beneficial effects on mucosal injury also appeared to be mediated 
by the presence of eNOS. In eNOS deficient mice, DPR with MEM was not able to protect the intestinal mucosa as well as in wildtype animals. It is difficult in this study to determine if DPR had beneficial effects directly on the intestine, or if these results were more likely mediated by the presence of better perfusion in wildtype animals. Given that the ablation of eNOS was confined to the endothelium in these knockouts, it is more likely that the preservation of histological architecture was due to improvements in postischemic mesenteric perfusion, and a more rapid influx of oxygen and vital nutrients in the post-injury phase.

The presence of worse hepatic inflammation in eNOSKO animals compared to wild type animals was also expected. There are ample data to suggest that the effects of intestinal ischemia not only affect the intestines, but also the liver [15]. Proinflammatory cytokines and other inflammatory mediators travel to the liver via portal blood flow following intestinal injury. Therefore, any inflammatory response seen in the intestines is likely to be observed in the liver as well. Herein, we saw that the presence of viable eNOS helped to decrease the inflammatory cascade within the liver. This phenomenon was supported by experiments showing less IL-1 $1 \beta$ and IL- 6 in the liver parenchyma when eNOS was intact. When eNOS was ablated, these inflammatory factors increased.

Although we expected to see higher levels of IL-1 $\beta$ and IL-6 in the intestinal tissue with eNOS ablation, we actually saw a decrease in IL-1 $1 \beta$ and no difference in IL-6 levels within the intestine following DPR with MEM. Although perplexing, it is certainly possible that endothelial nitric oxide pathways could actually promote increased IL-1 $\beta$ levels in the intestine. Several studies surrounding endotoxemia and sepsis have shown that IL$1 \beta$ promotes NO induced vasodilation [18]. Therefore, in the absence of eNOS, IL-1 $\beta$ may be downregulated due to a variety of negative feedback loops.

Even though IL-6 was expected to be elevated in these intestines, we did not 
observe a difference in WT and eNOS treated animals. Death of cells in intestinal tissue as a result of severe ischemic injury certainly activates inflammatory cascades, however this response has a temporal pattern. Cytokines increase at the onset of insult but as time passes they return to normal levels [19]. Our previous experience has shown that early tissue harvest and protein quantification leads to significant difference in cytokines in intestines of animals that undergone I/R injury [4]. However, we elected to increase the recovery time in these experiments to 48 hours in order to capture differences in mesenteric perfusion. In doing this, some of the acute phase reactants, including IL-1 $\beta$ and IL-6, may have normalized in certain tissues. However, this still does not explain why these factors continued to be elevated in the liver.

In addition to animal experiments, several human trials have demonstrated significant benefits with the utilization of DPR. Direct peritoneal resuscitation has been proven effective in resuscitation of brain dead cadaver organ donors during the periods prior to organ procurement [20]. These donors have impaired hormone physiology, an enhanced systemic inflammatory response, and end organ microcirculatory dysfunction. Brain dead donors who were treated with DPR had reduced IV fluid requirements and vasopressor requirements [20]. DPR has also been used in trauma patients that have undergone damage control surgery and has led to decreased time to abdominal closure, as well as reduction in intra-abdominal complications following damage control emergency surgeries [5].

\section{LIMITATIONS:}

The superior mesenteric artery ligation model of intestinal ischemia and reperfusion injury does not model clinical intestinal ischemia to its fullest. Although ischemia of the entire small bowel is possible secondary to SMA thrombus or embolus, 
the majority of intestinal ischemic episodes are due to segmental ischemia, such as may be seen with bowel obstructions or incarcerated hernias. Nonetheless, this model mimics the most severe form of intestinal ischemia, and therefore, is likely the best animal model available to test the effectiveness of new therapies.

\section{CONCLUSION:}

DPR has the potential to revolutionize the management of patients with acute intestinal ischemia. Although DPR has proven beneficial in a number of clinical scenarios, the choice of the dialysate during therapy is extremely important. In this study, we saw that DPR using minimal essential medium was able to improve postischemic recovery of intestinal blood flow, prevent mucosal injury, and alter the local inflammatory response in the intestine and liver. These beneficial effects appear to be mediated, in part, by endothelial nitric oxide pathways. Clinical trials designed to focus on DPR for patients with acute intestinal ischemia should therefore be explored.

\section{FIGURE LEGENDS:}

Figure 1: Direct peritoneal resuscitation improves mesenteric perfusion following ischemia and reperfusion injury through eNOS dependent pathways. (A) Following ischemia-reperfusion injury, Direct Peritoneal Resuscitation using Minimum Essential Medium (MEM) improves mesenteric perfusion in wild type mice. (B) In eNOSKO animals, no improvement in mesenteric perfusion was observed following DPR therapy with MEM. ${ }^{*}=p<0.05$ versus WT Vehicle

\section{Figure 2: Histological examination of the small intestine following $\mathrm{I} / \mathrm{R}$ injury and} therapy with DPR. (A) Histological analysis of intestinal tissue shows better 
preservation of intestinal architecture in wild type animals treated with MEM during DPR.

(B) eNOSKO animals treated with MEM during DPR did not have any beneficial increase in post-ischemic intestinal injury. ${ }^{*}=p<0.05$ versus WT Vehicle

\section{Figure 3: Intestinal Cytokines following Injury and Treatment with DPR. (A)} Intestinal concentrations of IL-1 $\beta$ were unexpectedly higher in WT animal intestines as compared to eNOSKO. (B) There were no significant differences in IL-6 levels between eNOSKO and WT treated MEM groups. ${ }^{*}=p<0.05$ versus WT

\section{Figure 4: Hepatic Cytokines following Injury and Treatment with DPR. Hepatic} concentrations of both (A) IL-1 $\beta$ and (B) IL-6 were significantly elevated following DPR with MEM therapy in eNOSKO animals as compared to WT. ${ }^{*}=p<0.05$ versus WT.

\section{REFERENCES:}

1. Heys, S.D., J. Brittenden, and T.J. Crofts, Acute mesenteric ischaemia: the continuing difficulty in early diagnosis. Postgrad Med J, 1993. 69(807): p. 4851.

2. Standring, S., et al., Gray's Anatomy: The anatomical basis of clinical practice. American Journal of Neuroradiology, 2005. 26(10): p. 2703.

3. Acosta-Merida, M.A., et al., Identification of risk factors for perioperative mortality in acute mesenteric ischemia. World J Surg, 2006. 30(8): p. 1579-85.

4. Crafts, T.D., et al., Direct peritoneal resuscitation improves survival and decreases inflammation after intestinal ischemia and reperfusion injury. journal of surgical research, 2015. 199(2): p. 428-434.

5. Smith, J.W., et al., Adjunctive treatment of abdominal catastrophes and sepsis with direct peritoneal resuscitation: indications for use in acute care surgery. Journal of Trauma and Acute Care Surgery, 2014. 77(3): p. 393-399.

6. Smith, J.W., et al., Direct peritoneal resuscitation improves inflammation, liver blood flow, and pulmonary edema in a rat model of acute brain death. J Am Coll Surg, 2014. 219(1): p. 79-87.

7. Zhang, J.J., et al., Protection against Intestinal Injury from Hemorrhagic Shock by Direct Peritoneal Resuscitation with Pyruvate in Rats. Shock, 2014.

8. Kalogeris, T., et al., Cell biology of ischemia/reperfusion injury. International review of cell and molecular biology, 2012. 298: p. 229. 
9. Altaany, Z., et al., The coordination of S-sulfhydration, $S$-nitrosylation, and phosphorylation of endothelial nitric oxide synthase by hydrogen sulfide. Sci. Signal., 2014. 7(342): p. ra87-ra87.

10. Craighead, D.H., et al., Mechanisms and time course of menthol-induced cutaneous vasodilation. Microvasc Res, 2017. 110: p. 43-47.

11. Cai, W., et al., Uric Acid Induces Endothelial Dysfunction by Activating the HMGB1/RAGE Signaling Pathway. Biomed Res Int, 2017. 2017: p. 4391920.

12. Chiu, C.-J., et al., Intestinal mucosal lesion in low-flow states: I. A morphological, hemodynamic, and metabolic reappraisal. Archives of surgery, 1970.101(4): p. 478-483.

13. Watkins, D.J., et al., Synergistic effects of HB-EGF and mesenchymal stem cells in a murine model of intestinal ischemia/reperfusion injury. Journal of pediatric surgery, 2013. 48(6): p. 1323-1329.

14. Maki, A.C., et al., Intestinal microcirculatory flow alterations in necrotizing enterocolitis are improved by direct peritoneal resuscitation. The American Surgeon, 2012. 78(7): p. 803-807.

15. Jensen, A.R., et al., Human adipose stromal cells increase survival and mesenteric perfusion following intestinal ischemia and reperfusion injury. Shock, 2016. 46(1): p. 75-82.

16. Jensen, A.R., et al., Harvest tissue source does not alter the protective power of stromal cell therapy after intestinal ischemia and reperfusion injury. J Surg Res, 2016. 204(2): p. 361-70.

17. Huang, A., et al., In eNOS knockout mice skeletal muscle arteriolar dilation to acetylcholine is mediated by EDHF. American Journal of Physiology-Heart and Circulatory Physiology, 2000. 278(3): p. H762-H768.

18. Bhagat, K., et al., Cytokine-induced venodilatation in humans in vivo: eNOS masquerading as iNOS. Cardiovasc Res, 1999. 41(3): p. 754-64.

19. la Garza, F.J.G.-d., et al., Temporal relationship of serum markers and tissue damage during acute intestinal ischemia/reperfusion. Clinics, 2013. 68(7): p. 1034-1038.

20. Smith, J.W., et al., Addition of direct peritoneal lavage to human cadaver organ donor resuscitation improves organ procurement. Journal of the American College of Surgeons, 2015. 220(4): p. 539-547. 


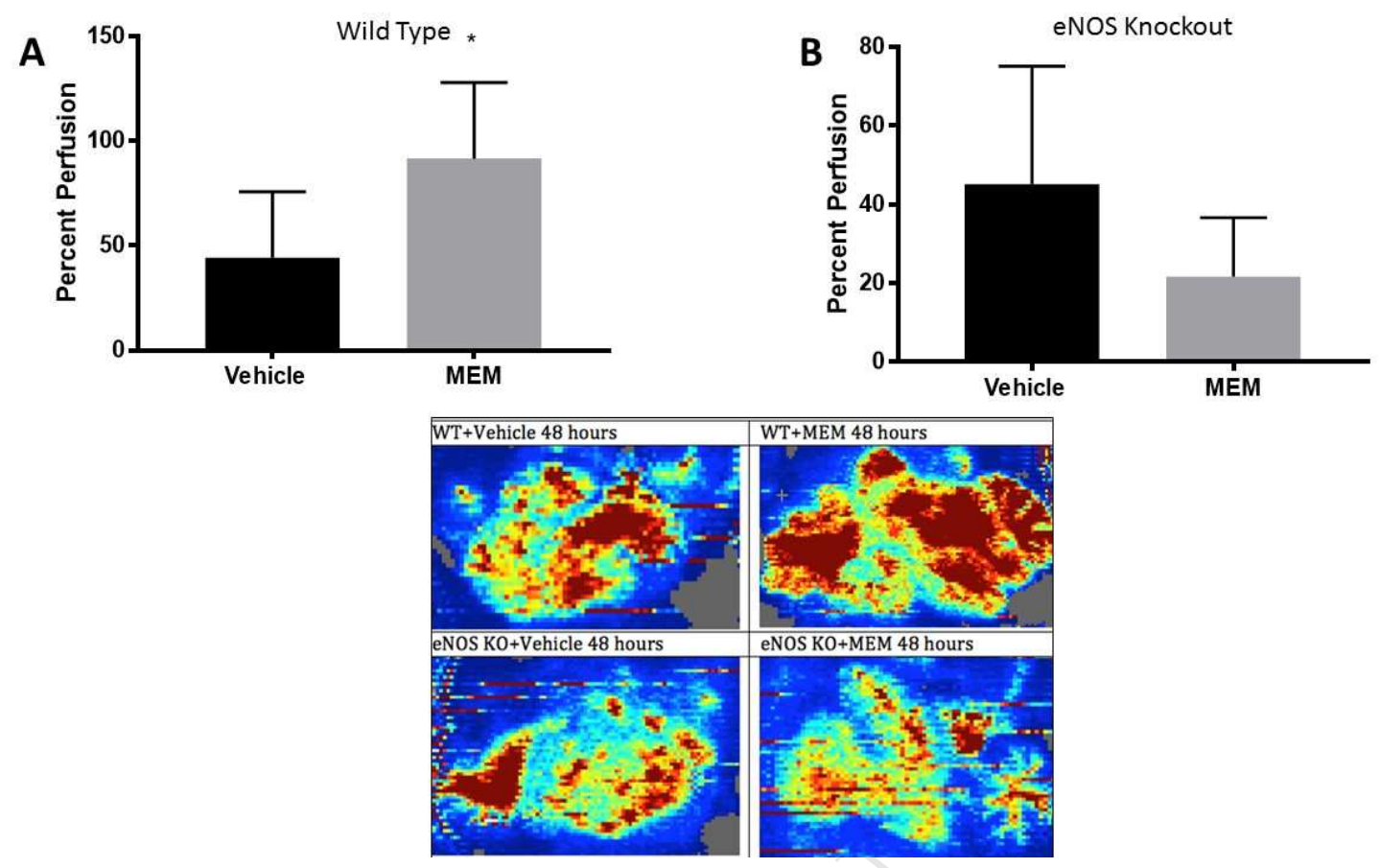




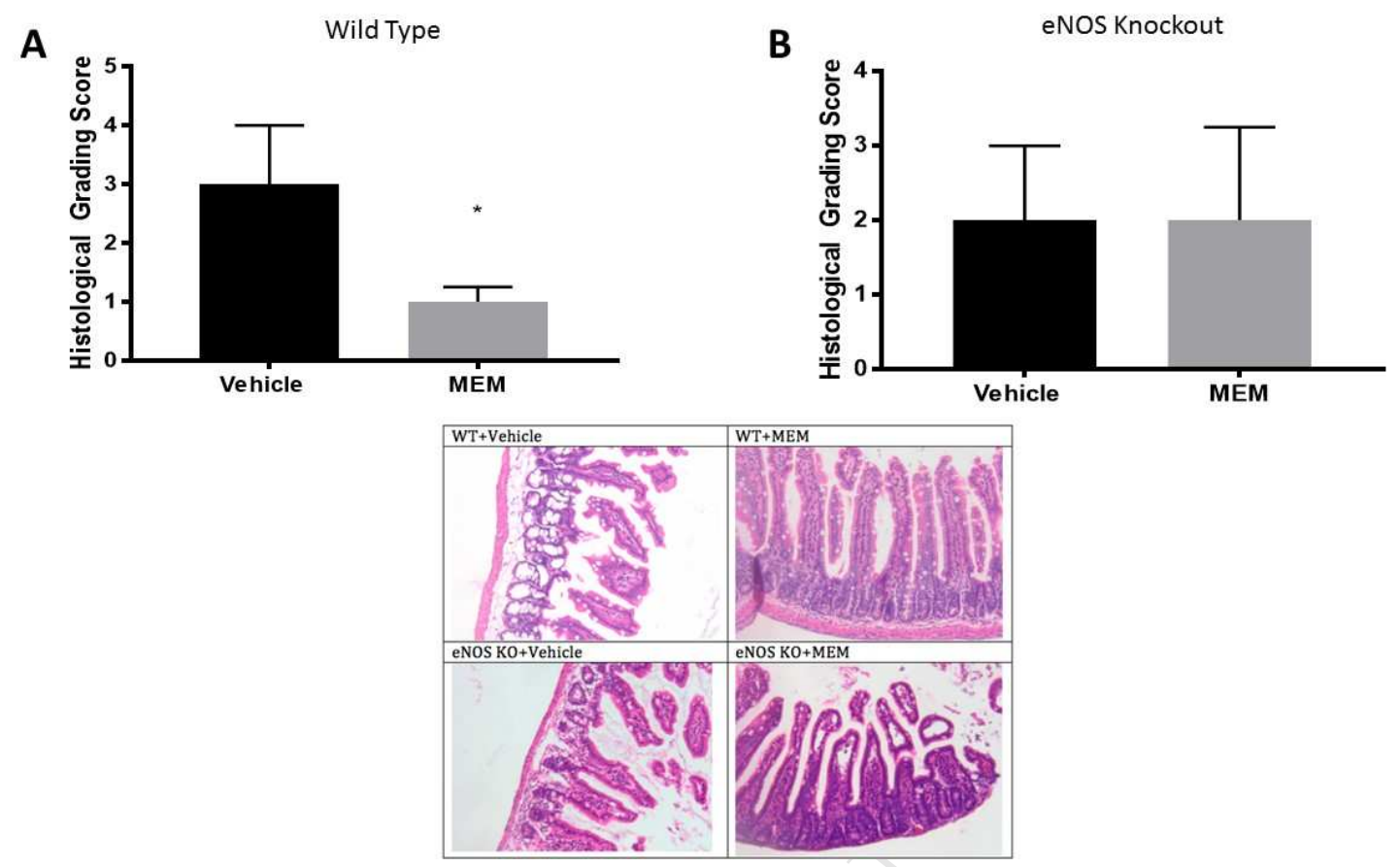


A

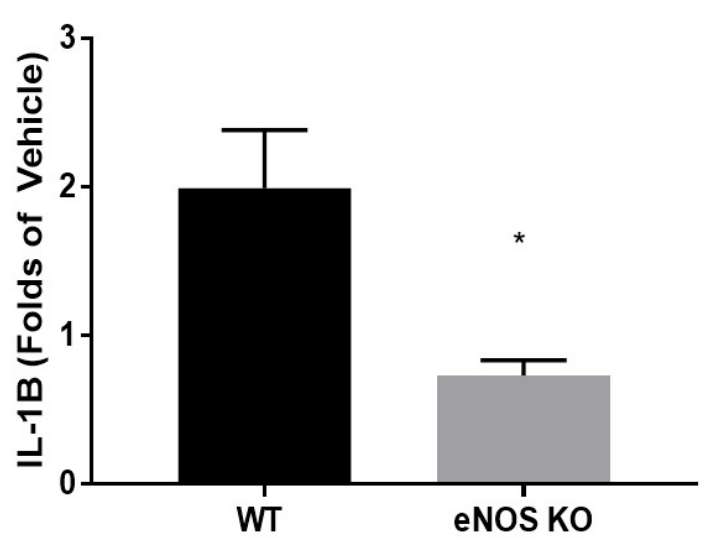

B

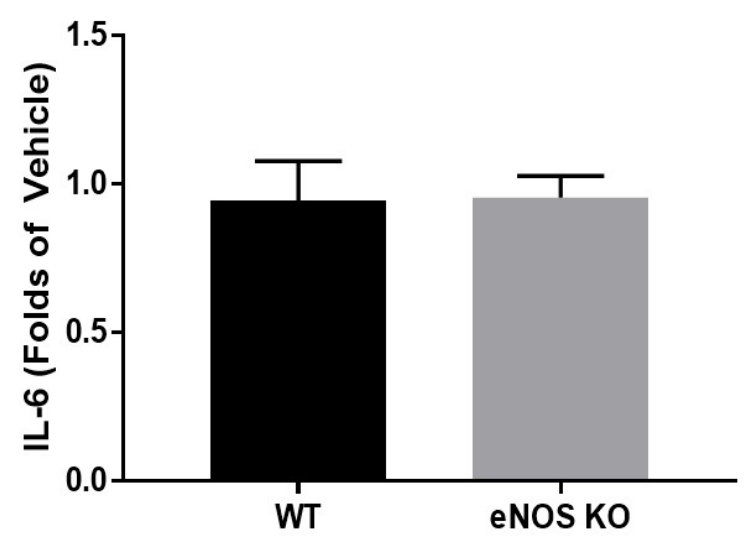


A

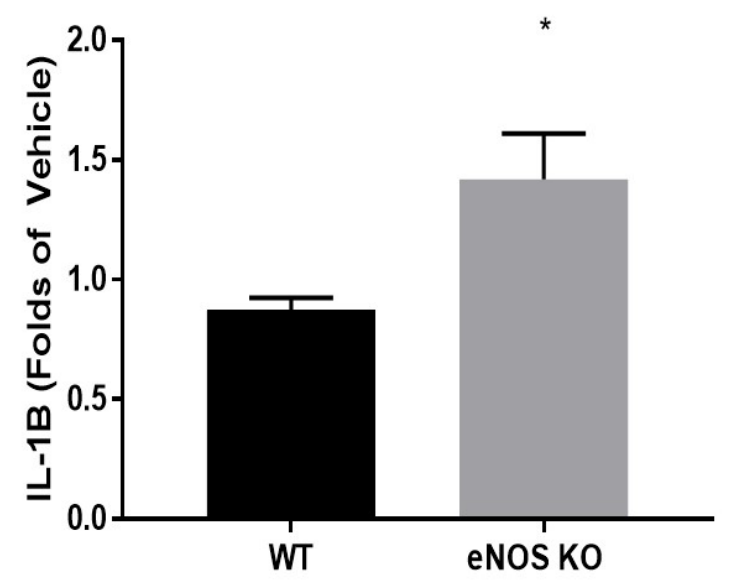

B

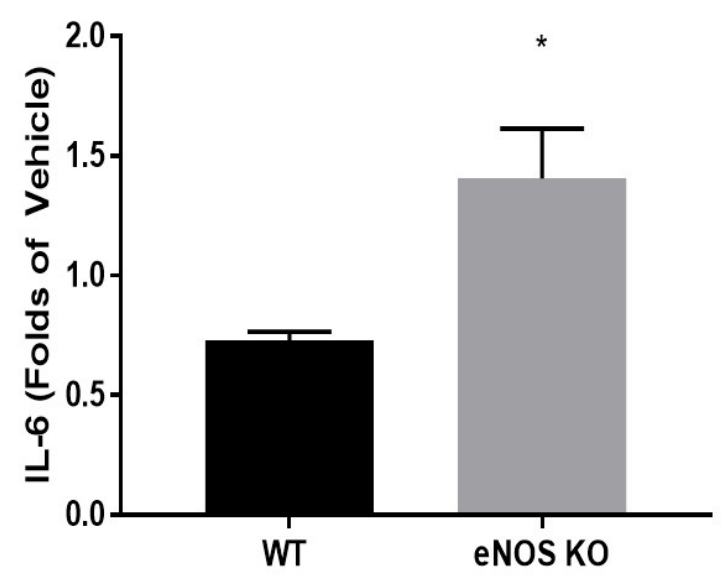

